\title{
Low-Loss SOI Photonic Wires and Ring Resonators Fabricated With Deep UV Lithography
}

\author{
Pieter Dumon, Student Member, IEEE, Wim Bogaerts, Student Member, IEEE, Vincent Wiaux, Johan Wouters, \\ Stephan Beckx, Joris Van Campenhout, Student Member, IEEE, Dirk Taillaert, Student Member, IEEE, \\ Bert Luyssaert, Student Member, IEEE, Peter Bienstman, Member, IEEE, Dries Van Thourhout, Member, IEEE, and \\ Roel Baets, Senior Member, IEEE
}

\begin{abstract}
We demonstrate single-mode photonic wires in Silicon-on-insulator with propagation loss as low as $2.4 \mathrm{~dB} / \mathrm{cm}$, fabricated with deep ultraviolet lithography and dry etching. We have also made compact racetrack and ring resonators functioning as add-drop filters, attaining $Q$ values larger than 3000 and low add-drop crosstalk.
\end{abstract}

Index Terms-High index contrast, photonic wire, ring resonators, Silicon-on-insulator (SOI) technology.

\section{INTRODUCTION}

$\mathbf{O}$ NE OF THE main requirements for the further integration of photonics is a low-loss waveguide that allows for short bends. This can be done using waveguides with a high refractive index contrast confining light by total internal reflection. In these so-called photonic wires, bend radii as small as $2 \mu \mathrm{m}$ are possible with low bend radiation losses. In such narrow high-index contrast waveguides, scattering at sidewall roughness is a severe cause of propagation loss [1]. Therefore, very accurate fabrication processes are needed. For research purposes, e-beam lithography is the preferred fabrication method. While this technique is very accurate, it is also slow. To fabricate photonic wires in Silicon-on-insulator (SOI), we opted for deep ultraviolet (DUV) lithography with an illumination wavelength of $248 \mathrm{~nm}$, as used for high-end complementary metal-oxide-semiconductor manufacturing [2], [3]. DUV lithography is better suited for mass fabrication, with a lower resolution, but offering large throughput and a large field size.

As sidewall roughness is a prime cause of propagation losses, good etching is required to keep the sidewalls as smooth as possible. Therefore, we did not etch the buried oxide cladding,

\footnotetext{
Manuscript received December 3, 2003; revised January 27, 2004. This work was supported in part by the IST-PICCO Project supported by the European Union and in the context of the Belgian IAP PHOTON Network. The work of P. Dumon, W. Bogaerts, and B. Luyssaert was supported by the Flemish Institute for the Industrial Advancement of Scientific and Technological Research (IWT) under a specialization grant. The work of J. Van Campenhout and P. Bienstman was supported by the Flemish Fund for Scientific Research (FWO-Vlaanderen) through a doctoral fellowship and postdoctoral fellowship, respectively. The work of D. Van Thourhout was supported by the Belgian Federal Office for Scientific, Technical and Cultural Affairs.

P. Dumon, W. Bogaerts, J. Van Campenhout, D. Taillaert, B. Luyssaert, P. Bienstman, D. Van Thourhout, and R. Baets are with the Photonics Research Group of the Department of Information Technology (INTEC), Ghent University-IMEC, 9000 Gent, Belgium (e-mail: Pieter.Dumon@ intec.UGent.be).

V. Wiaux, J. Wouters, and S. Beckx are with the Silicon Processing Technology Division of IMEC vzw, 3001 Leuven, Belgium.

Digital Object Identifier 10.1109/LPT.2004.826025
}

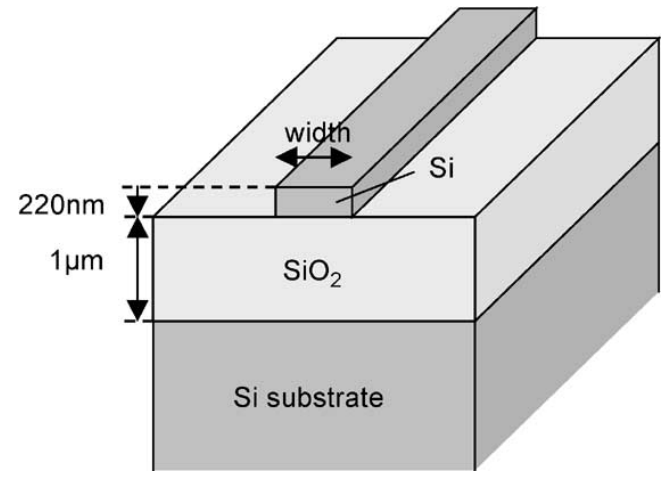

Fig. 1. SOI photonic wire waveguide. For single-mode operation, the wire width should be less than $600 \mathrm{~nm}$.

but only the top Silicon layer. This structure is illustrated in Fig. 1. No additional roughness reduction like thermal oxidation [4] was applied. We measured propagation losses as low as $2.4 \mathrm{~dB} / \mathrm{cm}$ for a $500-\mathrm{nm}$-wide wire. We have made ring resonators and racetrack resonators with the same technology, attaining $Q$ values of over 3000 .

\section{Straight WaVeguides}

The photonic wire illustrated in Fig. 1 is single mode up to a width of about $600 \mathrm{~nm}$. The transverse electrice (TE) and transverse magnetic (TM) modes have very different effective index and propagation loss. Polarization independency is very hard to achieve, although it is possible to change this by altering the core form factor.

The propagation loss in single-mode photonic wires in SOI is caused by substrate leakage and scattering at sidewall roughness. In an SOI wire, only leaky modes exist. Although the confinement of the mode in the core is very good, the exponential tail of the mode still extends into the substrate, causing substrate leakage. This loss decreases exponentially with increasing oxide thickness. Also, as making the waveguide smaller decreases confinement, substrate leakage is larger with smaller waveguides. We calculated the substrate leakage loss using a fully vectorial mode solver with perfectly matched layer boundary conditions [5]. Fig. 2 shows the substrate leakage versus oxide thickness for different widths for TE modes. With 1- $\mu$ m oxide, losses are acceptable. Substrate leakage is much higher for TM polarization.

Line-edge roughness (LER) causes scattering loss. The cause of sidewall roughness can be found in both lithography [6], re- 


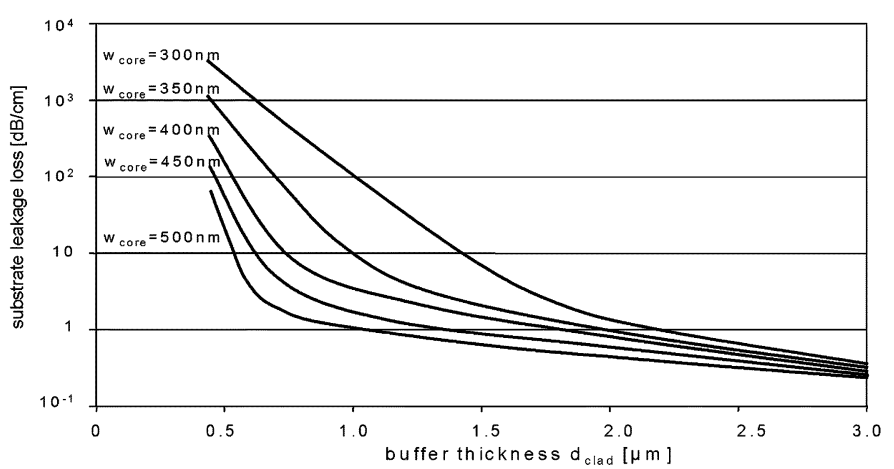

Fig. 2. Substrate leakage of a photonic wire versus oxide thickness, for several widths, as calculated with mode expansion software [5] (TE).

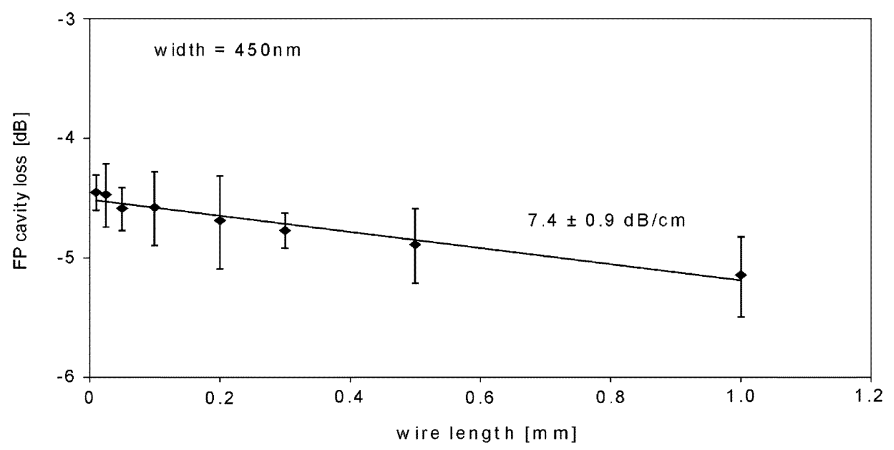

Fig. 3. Total Fabry-Pérot cavity loss for cavities with 450-nm-wide wires of different lengths at $1550-\mathrm{nm}$ wavelength (TE polarization). The wire loss can be extracted from the slope of the fitted line. The sum of facet reflection and loss of the tapers can be calculated from the intercept.

sist chemistry [7], and etching. The exact causes are still under debate, however. The etching transfers the LER already present in the resist pattern to the etched material while adding new roughness. Generally, etching deeper will result in more roughness. Therefore, we only etched the silicon top layer.

To characterize the waveguide losses we measured the transmission spectrum of the cavity formed by two cleaved facets in a 3- $\mu \mathrm{m}$-wide ridge waveguide. In the middle of the cavity, the waveguide was tapered down to a photonic wire using an adiabatic taper of 300- $\mu \mathrm{m}$ length. We measured various cavities with different lengths of photonic wires, up to $1 \mathrm{~mm}$. We extracted the total cavity loss from the peak-to-valley ratio of the resulting Fabry-Pérot transmission spectrum. This cavity loss contains both the waveguide loss and the losses from "lumped elements," like the tapers and the facets. When plotted on a logarithmic scale as a function of wire length, the propagation loss of the photonic wires can be extracted from the slope of the fitted straight line, as can be seen in Fig. 3 for a wire of 450-nm width.

We measured the propagation loss for wires of various lengths for TE polarization, and we see that the losses decrease exponentially for broader wires. This is in agreement with sidewall roughness and substrate leakage as the loss mechanisms. For a 500-nm wire, which is still a single-mode waveguide, we have measured a propagation loss of $2.4 \mathrm{~dB} / \mathrm{cm}$. Fig. 2 shows that theoretically, $1-1.5 \mathrm{~dB} / \mathrm{cm}$ of this is due to substrate leakage. Scattering losses are clearly very low.

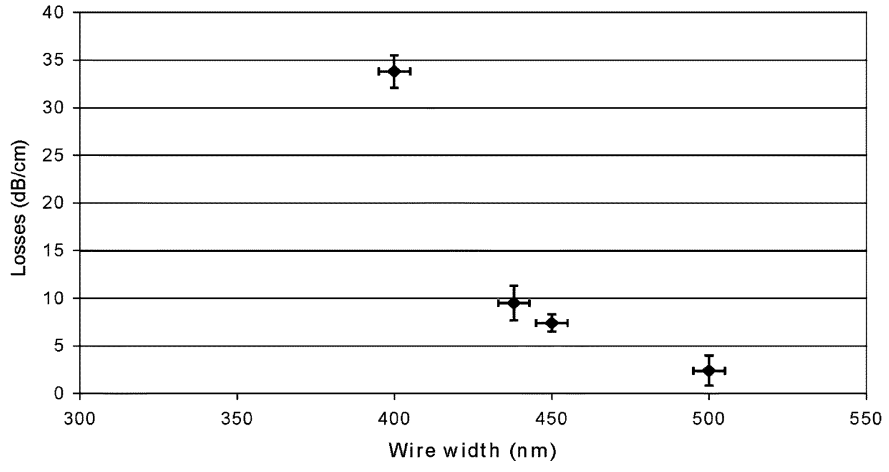

Fig. 4. Propagation losses at 1550-nm wavelength for SOI photonic wires as a function of wire width (TE polarization).

TABLE I

SOI WAVEGUIDE LOSSES

\begin{tabular}{crrlrr}
\hline \hline Waveguide width & \multicolumn{3}{c}{ Propagation loss } & \multicolumn{2}{c}{$\begin{array}{c}\text { Loss per taper } \\
\text { (upper estimate) }\end{array}$} \\
\hline $400 \mathrm{~nm}$ & 33.8 & \pm 1.7 & $\mathrm{~dB} / \mathrm{cm}$ & 0.27 & $\mathrm{~dB}$ \\
$440 \mathrm{~nm}$ & 9.5 & \pm 1.8 & $\mathrm{~dB} / \mathrm{cm}$ & 0.23 & $\mathrm{~dB}$ \\
$450 \mathrm{~nm}$ & 7.4 & \pm 0.9 & $\mathrm{~dB} / \mathrm{cm}$ & 0.22 & $\mathrm{~dB}$ \\
$500 \mathrm{~nm}$ & 2.4 & \pm 1.6 & $\mathrm{~dB} / \mathrm{cm}$ & 0.19 & $\mathrm{~dB}$ \\
\hline \hline
\end{tabular}

Propagation losses at 1550-nm wavelength for SOI photonic wires of various widths. (TE polarization.) The values are also plotted in Fig. 4. An upper boundary for the taper loss is calculated by subtracting the theoretical facet loss from the intercept of the fitted line in Fig. 3.

From the total loss of the lumped elements given by the intercept of the fitted line and the vertical axis in Fig. 3, we estimated the taper losses. The facet reflection is assumed to be identical for all 3- $\mu$ m-wide ridges, with a value of $39.30 \%$ as calculated with mode expansion software. This gives an upper boundary of $0.19 \mathrm{~dB}$ for the loss of a $300-\mu \mathrm{m}$-long taper from 3- $\mu \mathrm{m}$ width down to a 500 -nm-wide wire.

Fig. 4 and Table I show the wire propagation losses in decibels/centimeters as function of the wire width, as well as the upper boundary for the taper losses. The increase in propagation loss for narrower wires is stronger than can be explained by the electric field strength of the guided mode on the silicon-air interface and substrate leakage. This implies that the sidewalls of narrow wires have more roughness than those of wider wires. SEM inspection of the structures confirms this.

\section{RING RESONATORS}

Resonators can provide building blocks for a large number of functional components on a photonic integrated circuit. In photonic wires, ring resonators are the easiest to implement. An example of a compact racetrack resonator is illustrated in Fig. 5. Here, the coupling between the straight wire and the resonator is enhanced by adding a straight coupling section. The resonator is symmetrically coupled to both waveguides.

Fig. 6 shows its pass and drop transmission spectra, normalized on the maximum pass port transmission. The resonator has a free-spectral range (FSR) of $14 \mathrm{~nm}$ and a 3-dB bandwidth of $0.5 \mathrm{~nm}$, leading to a finesse of 28 or a $Q$ factor over 3000 . By fitting the theoretical model to the measured data we extracted the group index in the ring, the coupling factor and the propagation loss in the cavity waveguide. 


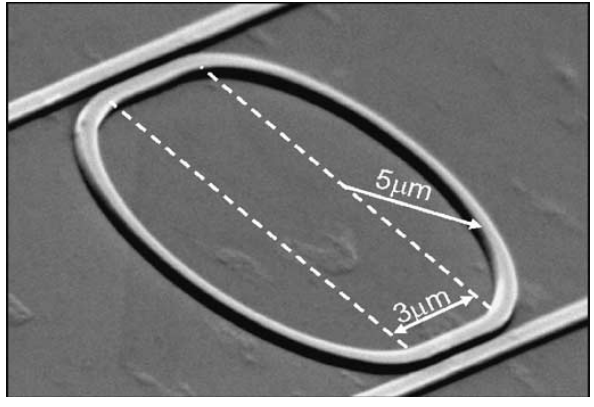

Fig. 5. Racetrack resonator in SOI. The isolated wire width is $500 \mathrm{~nm}$, the gap width is $230 \mathrm{~nm}$. Note that when the wires get closer together, the wire width decreases to $450 \mathrm{~nm}$ due to optical proximity effects during lithography [3].

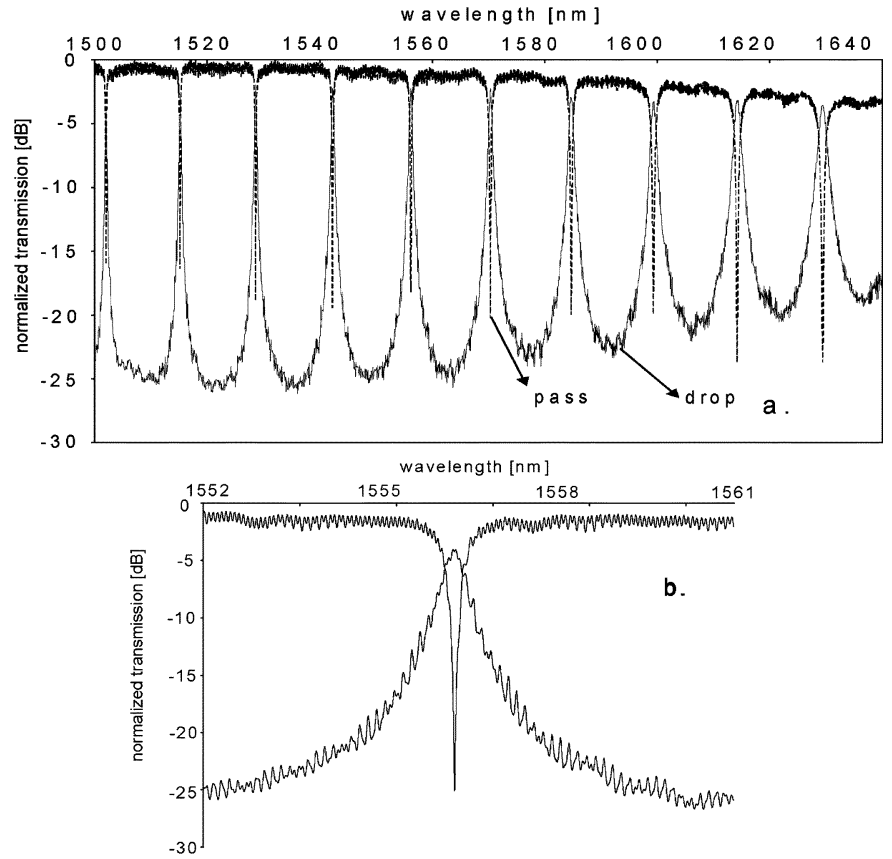

Fig. 6. (a) Pass and drop transmission spectra of the racetrack resonator from Fig. 5. (b) Detail, with $Q$ factor over 3000, the fluctuations are due to the nonoptimal antireflective coating of the sample facets. The spectra are normalized on the maximum pass port transmission. (TE polarization.)

For this resonator, the extracted cavity waveguide group index is around 4.6, showing large dispersion as the effective mode index is about 2.5. This group index is a mean value over the ring, because in the coupling region, the wire is narrower due to optical proximity effects [3] and the group index in the straight sections is different from that in the bends. It is well-known that for small resonators, the resonance wavelength is extremely intolerant to fabrication variations.

We measured group index changes around $1 \%$ with a waveguide width change of about $5 \mathrm{~nm}$, leading to a shift in resonance wavelength of around $15 \mathrm{~nm}$. A method to tightly control the optical length of the cavity is clearly needed.

The racetrack form factor performs well: With a ring resonator, the amplitude coupling varies from 0.1 to 0.2 , leading to high $Q$ (8000 measured) but high add-drop crosstalk. The racetrack resonators have a coupling factor of 0.3 around 1550-nm wavelength, which is fairly optimal. Add-drop crosstalk reaches
$-20 \mathrm{~dB}$, the difference between the throughput at resonance and the minimum off-resonance crosstalk reaches $-25 \mathrm{~dB}$. Insertion loss to the drop port is around $3 \mathrm{~dB}$.

For a racetrack resonator with $5-\mu \mathrm{m}$ radius and $3-\mu \mathrm{m}$ straight sections, 450-nm width of the bent wire and 415-nm width of the coupler waveguides, the fitted total roundtrip loss (not including coupling) around $1550-\mathrm{nm}$ wavelength is $0.2 \mathrm{~dB}$, or $5 \mathrm{~dB} / \mathrm{mm}$. With 450- and 415-nm-wide straight wires having propagation losses of 0.74 and $2.2 \mathrm{~dB} / \mathrm{mm}$, respectively, the bent and straight sections clearly introduce extra loss mechanisms in the cavity. Simulation with mode expansion software shows that due to the bending, the mode profile changes and the field strength in the exponential mode tail in the substrate is higher, leading to higher substrate leakage. Simulations show that the intrinsic bending loss which theoretically would also occur without substrate is negligible. There is some additional loss due to the mismatch between the straight and bent sections in a racetrack resonator. These loss figures limit the achievable $Q$ factor and add-drop crosstalk level. If necessary, the add-drop crosstalk could be further reduced by trading for $Q$ factor by using asymmetric coupling [8]. Still, the performance of the resonator is already good enough for real-life use over a wavelength range of a few times the FSR when the ring's group index can be controlled.

\section{CONCLUSION}

We have demonstrated single-mode photonic wires in SOI fabricated with DUV lithography. The measurements show propagation loss as low as $2.4 \mathrm{~dB} / \mathrm{cm}$ for a $500-\mathrm{nm}$-wide waveguide. We demonstrated racetrack resonators with $Q$ factors over 3000 and low crosstalk and insertion loss figures illustrating the capabilities of photonic wire technology.

\section{ACKNOWLEDGMENT}

The authors would like to thank D. Vangoidsenhoven for the wafer exposures and R. de Ruyter and J. Mees for their work on the mask design.

\section{REFERENCES}

[1] W. Bogaerts, P. Bienstman, and R. Baets, "Scattering at sidewall roughness in photonic crystal slabs," Opt. Lett., vol. 28, no. 9, pp. 689-691, 2003.

[2] W. Bogaerts et al., "Large-scale production techniques for photonic nanostructures," Proc. SPIE, vol. 5225, pp. 101-112, 2003.

[3] — , "Fabrication of photonic crystals in Silicon-on-insulator using 248-nm deep UV lithography," IEEE J. Select. Topics Quantum Electron., vol. 8, pp. 928-934, July/Aug. 2002.

[4] K. K. Lee et al., "Fabrication of ultralow-loss $\mathrm{Si} \mathrm{SiO}_{2}$ waveguides by roughness reduction," Opt. Lett., vol. 26, no. 23, pp. 1888-1890, 2001.

[5] P. Bienstman and R. Baets, "Optical modeling of photonic crystals and VCSELs using eigenmode expansion and perfectly matched layers," Opt. Quantum El., vol. 33, pp. 327-341, Jan. 2001.

[6] M. Williamson and A. Neureuther, "Enhanced quantitative analysis of resist image contrast upon line-edge roughness (LER)," Proc. SPIE, vol. 5039, pp. 423-432, June 2003.

[7] H. Fukuda, "Analysis of line edge roughness using probability process model for chemically amplified resists," Jpn. J. Appl. Phys., vol. 42, no. 6B, pp. 3748-3754, June 2003.

[8] A. Vörckel et al., "Asymmetrically coupled Silicon-on-insulator microring resonators for compact add-drop multiplexers," IEEE Photon. Technol. Lett., vol. 15, pp. 921-923, July 2003. 